\title{
Evaluating increased resource use in fibromyalgia using electronic health records
}

\author{
Jay M Margolis' \\ Elizabeth T Masters ${ }^{2}$ \\ Joseph C Cappelleri ${ }^{3}$ \\ David M Smith' \\ Steven Faulkner ${ }^{4}$
}

'Truven Health Analytics, Life Sciences, Outcomes Research, Bethesda, MD, ${ }^{2}$ Pfizer Inc, Outcomes \& Evidence, New York, NY, ${ }^{3}$ ffizer Inc, Statistics, Groton, CT, ${ }^{4}$ Pfizer Inc, North American Medical Affairs, Medical Outcomes Specialists, St Louis, MO, USA
Correspondence: Jay M Margolis Truven Health Analytics, 332 Bryn Mawr Avenue, Bala Cynwyd, PA 19004, USA

$\mathrm{Tel}+\mathrm{I} 6106674718$

Fax +16106674718

Email jay.margolis@truvenhealth.com
This article was published in the following Dove Press journal:

ClinicoEconomics and Outcomes Research

16 November 2016

Number of times this article has been viewed

Objective: The management of fibromyalgia (FM), a chronic musculoskeletal disease, remains challenging, and patients with FM are often characterized by high health care resource utilization. This study sought to explore potential drivers of all-cause health care resource utilization and other factors associated with high resource use, using a large electronic health records (EHR) database to explore data from patients diagnosed with FM.

Methods: This was a retrospective analysis of de-identified EHR data from the Humedica database. Adults ( $\geq 18$ years) with FM were identified based on $\geq 2$ International Classification of Diseases, Ninth Revision codes for FM (729.1) $\geq 30$ days apart between January 1, 2008 and December 31, 2012 and were required to have evidence of $\geq 12$ months continuous care pre- and post-index; first FM diagnosis was the index event; 12-month pre- and post-index reporting periods. Multivariable analysis evaluated relationships between variables and resource utilization.

Results: Patients were predominantly female (81.4\%), Caucasian $(87.7 \%)$, with a mean (standard deviation) age of 54.4 (14.8) years. The highest health care resource utilization was observed for the categories of "medication orders" and "physician office visits," with 12-month post-index means of 21.2 (21.5) drug orders/patient and 15.1 (18.1) office visits/patient; the latter accounted for $73.3 \%$ of all health care visits. Opioids were the most common prescription medication, $44.3 \%$ of all patients. The chance of high resource use was significantly increased $(P<0.001) 26 \%$ among African-Americans vs Caucasians and for patients with specific comorbid conditions ranging from $6 \%$ (musculoskeletal pain or depression/bipolar disorder) to $21 \%$ (congestive heart failure). Factors significantly associated with increased medications ordered included being female $(P<0.001)$ and specific comorbid conditions $(P<0.05)$.

Conclusion: Physician office visits and pharmacotherapy orders were key drivers of all-cause health care utilization, with demographic factors, opioid use, and specific comorbidities associated with resource intensity. Health systems and providers may find their EHRs to be a useful tool for identifying and managing resource-intensive FM patients.

Keywords: fibromyalgia, electronic health records, health care resource utilization

\section{Introduction}

Fibromyalgia (FM) is a chronic musculoskeletal disease with evidence suggesting that it results from a dysfunction of central pain processing pathways. ${ }^{1,2}$ The clinical characteristics of FM include the hallmark symptom of chronic widespread pain, which is a key feature required for diagnosis, ${ }^{3}$ as well as a constellation of other symptoms such as sleep disturbances, headaches, fatigue, reduced function, anxiety, and depression. ${ }^{4}$ The prevalence of FM in the US has been estimated to be between $\sim 2 \%$ and $6 \%,{ }^{5,6}$ with middle-aged women disproportionately affected.? 
Management of FM remains challenging despite the development of guidelines that recommend use of several medication classes ${ }^{8,9}$ and the approval by the US Food and Drug Administration of three medications indicated for its treatment (duloxetine, milnacipran, and pregabalin). While opioids have been consistently reported to be one of the most commonly prescribed pain-related medications in patients with $\mathrm{FM},{ }^{2,10-14}$ they are neither effective nor recommended for long-term management, ${ }^{15,16}$ with only weak opioids such as tramadol suggested in guidelines. ${ }^{8,9,17}$ There is evidence suggesting that prescribing of opioids is also associated with a lower likelihood of being prescribed guideline-recommended FM medications. ${ }^{2}$

In addition to the patient burden associated with FM resulting from impaired function, productivity, and quality of life, ${ }^{18-21}$ health care resource utilization is significantly higher in patients with FM than among non-FM controls. ${ }^{22,23}$ This greater utilization has a substantial economic impact that also appears to be greater as FM severity increases. ${ }^{24}$ Identifying drivers of health care resource utilization in patients with FM and factors associated with this use can inform development of strategies to more efficiently manage these patients. The emergence and implementation of electronic health records (EHR) provides an opportunity to explore how these data can be used to evaluate and understand resource utilization in patients with FM. ${ }^{23-26}$ Such an understanding may provide a first step in developing strategies to improve health care for patients with FM and reduce costs.

EHRs capture longitudinal, patient-level clinical data that represent integral components of provider care, allowing more complete evaluation of a wider range of variables than would be available in health care claims or other observational data sources. ${ }^{25}$ Included in EHRs are textual data not associated with a diagnosis or other coding, which can be recorded as structured data (e.g., physical assessments) or as unstructured text. ${ }^{25}$ Thus, an additional strength of EHR is this ability to capture unstructured information in the notes fields, and since many symptoms attributable to FM, such as sleep disturbances, fatigue, and headache, are not necessarily represented in claims, reports of these symptoms can be harvested from the unstructured data. ${ }^{23,25,26}$ While integration of EHR into the management of FM has previously been suggested as a means to improve patient care, ${ }^{26}$ only two published studies have applied this technology to FM, both with the purpose of better understanding factors associated with a diagnosis..$^{23,27}$ Therefore, the purpose of this study was to identify potential drivers of all-cause health care resource utilization and characterize factors associated with high resource use, using a large EHR database to explore data from patients diagnosed with FM.

\section{Methods \\ Data source}

This retrospective analysis used structured and unstructured data from the de-identified Humedica EHR database, which has broad representation across all geographic regions of the US. The database longitudinally captures data from hospitals, medical groups, and integrated delivery networks, including information on demographics, diagnoses, and a complete range of inpatient and outpatient encounters as well as medications ordered. Humedica aggregates de-identified EHR data from providers across the continuum of care. Records are linked using a unique patient identifier and are fully compliant with the Health Insurance Portability and Accountability Act (HIPAA). The database was certified to satisfy conditions set forth in Sections 164.514 (a)-(b)1ii of the HIPAA privacy rule regarding the determination and documentation of statistically de-identified data. Therefore because this study used only de-identified patient records and did not involve the collection, use, or transmittal of individually identifiable data, Institutional Review Board (IRB) approval to conduct this study was not required.

The structured data include demographic information, clinical characteristics, and health care resource utilization. Unstructured data were searched for information on FM pain symptoms using Natural Language Processing (NLP) techniques. The Humedica NLP system was developed using vocabulary from the Unified Medical Language System that includes multiple medical dictionaries such as the Logical Observation Identifiers Names and Codes and the Systemized Nomenclature of Medicine-Clinical Terms. These data are harvested from the notes fields within the EHR, and each NLP concept included in the data is associated with a unique identifier and a date of observation, which also allows longitudinal tracking.

\section{Population}

The evaluated population consisted of adults ( $\geq 18$ years at index) identified with FM based on $\geq 2$ International Classification of Diseases, Ninth Revision codes for FM (729.1) $\geq 30$ days apart between January 1, 2008 and December 31, 2012 and who had $\geq 12$ months continuous care pre- and post-index, with continuous care determined using the "first month active" and "last month active" fields, reflecting the first and most recent months with recorded health care activity events in the Humedica data set. The first reported FM diagnosis was the index event. Patients were excluded if they had evidence during pre- or post-index study periods of a diagnosis, procedure, or an indication of transplant surgery or cancer, except for basal cell or squamous cell skin cancers and benign neoplasms. 


\section{Outcomes and analyses}

All-cause health care resource utilization during the 12-month post-index period was examined using descriptive statistics (proportions of patients and units of resource) for the resource categories of inpatient admissions, emergency room, medications ordered, physician office visits, physical therapy, radiology, laboratory, and other outpatient visits (ie, excludes physician office visits, radiology, laboratory, and emergency room). For evaluation of medications, pharmacotherapy use was defined as evidence of a National Drug Code on an outpatient prescription order. The medication may have been prescribed for any of the patient's conditions, including but not limited to FM. Evaluation of opioid use was also stratified as those mentioned in FM guidelines (weak opioids including tramadol, codeine and codeine combinations, and tapentadol) and those not recommended in guidelines. ${ }^{8,9,17}$ Comorbidities and clinical symptoms, including those reported in prior FM studies, were identified during the pre- and post-index study periods from evidence of an International Classification of Diseases, Ninth Revision, Clinical Modification diagnosis code (for conditions where codes were available) or a positive mention of the comorbidity in the unstructured text of the EHR, and grouped according to categories and nomenclature used in prior FM studies. ${ }^{3,47,28}$

Multivariable analyses using generalized linear models $(\mathrm{GLMs})^{29}$ were performed with demographic, clinical, and pharmacotherapy factors as covariates to determine how these characteristics related to high resource utilization. For analyzing the number of visits and number of medications, which were counts, negative binomial regression generalized linear modeling with a negative binomial distribution and log link was used. Negative binomial regressions, rather than Poisson regressions, were used because overdispersion of the counts compared with the Poisson is usual with this type of data. Overdispersion was confirmed by examination of scale factors based on the ratio of observed variances compared with those expected under a Poisson model ie, the means. To identify those covariates showing significance, a stepwise regression approach with forward selection was used with $P$-values of 0.05 for both inclusion and removal of covariates from the model. The level of statistical significance for all statistical tests was 0.05 . All statistical analyses were conducted using SAS version 9.4 (SAS Institute Inc., Cary, NC, USA).

\section{Results}

Of 265,341 subjects with a diagnosis of FM between January 1, 2008 and December 31, 2012, a total of 64,038 met all other criteria for inclusion in the analysis. This population was primarily female (81.4\%) and Caucasian (87.7\%), with a mean
Table I Demographic characteristics $(\mathrm{N}=64,038)$

\begin{tabular}{ll}
\hline Variable & Value \\
\hline Age, years, mean (SD) & $54.4(14.8)$ \\
Age distribution, $\mathbf{n}$ (\%; years) & \\
I8-34 & $16,079(25.1)$ \\
$35-44$ & $15,680(24.5)$ \\
$45-54$ & $15,507(24.2)$ \\
$55-64$ & $13,531(21.1)$ \\
$\geq 65$ & $3,241(5.1)$ \\
Female, n (\%) & $52,108(81.4)$ \\
Ethnicity, n (\%) & \\
Caucasian & $56,145(87.7)$ \\
Black/African-American & $4,529(7.1)$ \\
Asian & $373(0.6)$ \\
Other & $2,991(4.7)$ \\
US geographic region, n (\%) & \\
Northeast & $2,987(4.7)$ \\
Midwest & $35,119(54.8)$ \\
South & $22,601(35.3)$ \\
West & $1,889(2.9)$ \\
Other & $1,442(2.3)$ \\
\hline Abreviation &
\end{tabular}

Abbreviation: SD, standard deviation.

Table 2 Clinical characteristics $(\mathrm{N}=64,038)$

\begin{tabular}{lll}
\hline $\begin{array}{l}\text { Comorbidities or symptoms } \\
\text { with a prevalence } \geq \mathbf{I} \text { \% in the } \\
\text { I2-month pre-index period, } \mathbf{n}(\%)\end{array}$ & Pre-index & Post-index \\
\hline Hypertension & $25,073(39.2)$ & $33,941(53.0)$ \\
Hyperlipidemia & $21,546(33.6)$ & $30,194(47.2)$ \\
Arthritis and other arthropathies & $21,525(33.6)$ & $33,685(52.6)$ \\
Nausea, vomiting & $19,782(30.9)$ & $29,096(45.4)$ \\
Depression & $19,017(29.7)$ & $29,966(46.8)$ \\
Gastroesophageal reflux disease & $17,532(27.4)$ & $26,883(42.0)$ \\
Rheumatism, excluding the back & $16,290(25.4)$ & $26,944(42.1)$ \\
Low back (lumbar) pain & $16,094(25.1)$ & $25,979(40.6)$ \\
Paresthesias, numbness, tingling & $15,304(23.9)$ & $24,006(37.5)$ \\
Diarrhea & $15,183(23.7)$ & $23,197(36.2)$ \\
Dizziness or balance problems & $14,979(23.4)$ & $23,677(37.0)$ \\
Other gastrointestinal conditions & $14,170(22.1)$ & $21,400(33.4)$ \\
Anxiety & $13,437(21.0)$ & $21,588(33.7)$ \\
Constipation & $12,077(18.9)$ & $19,535(30.5)$ \\
Back or neck pain (non-lumbar) & $11,963(18.7)$ & $20,077(31.4)$ \\
Osteoarthritis & $11,533(18.0)$ & $20,718(32.4)$ \\
Abdominal pain/cramps & $10,181(15.9)$ & $15,229(23.8)$ \\
Other musculoskeletal pain & $9,983(15.6)$ & $16,939(26.5)$ \\
Insomnia, sleep disorders & $8,085(12.6)$ & $16,204(25.3)$ \\
Migraine, headache & $7,929(12.4)$ & $12,439(19.4)$ \\
Cerebrovascular disease & $7,734(12.1)$ & $12,432(19.4)$ \\
Muscle stiffness, rigidity, tenderness & $6,573(10.3)$ & $12,015(18.8)$ \\
\hline
\end{tabular}

(standard deviation [SD]) age of 54.4 (14.8) years (Table 1). Comorbidities representing a variety of systems were present in substantial proportions of patients (Table 2), with hypertension (39.2\%), hyperlipidemia (33.6\%), arthritis/other arthropathies (33.6\%), nausea/vomiting (30.9\%), and depression (29.7\%) the most frequent conditions pre-index. Similarly, these were the most common comorbidities post-index, although reported 
in higher percentages of patients (hypertension $(53.0 \%)$, hyperlipidemia (47.2\%), arthritis/other arthropathies (52.6\%), nausea/vomiting (45.4\%), and depression (46.8\%)) (Table 2).

The mean (SD) pain score (pain scales were scored $0=$ no pain to $10=$ worst pain; the data source did not identify names of specific pain scales used) was 4.5 (3.9), but pain scores, which were taken from the structured data, were only available for 2,321 patients $(3.6 \%)$ during the pre-index period. Among the

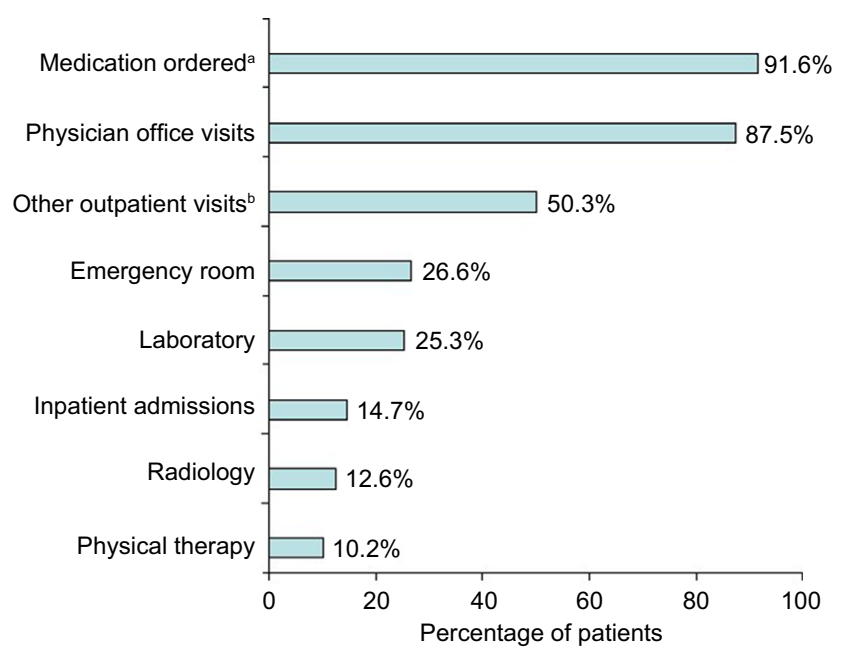

Figure I Proportion of patients using health care resource categories during the I2-month post-index period among patients with fibromyalgia.

Notes: aMedication orders regardless of condition. 'Outpatient visits other than physician office visits, emergency room, radiology, and laboratory visits.
$19,350(30.2 \%)$ patients for whom pain scores could be identified in the post-index period, the mean (SD) score was 3.9 (4.8).

As shown in Figure 1, the health care resource categories used by the highest proportion of patients during the 12-month post-index period, were "medications ordered" (91.6\%) and "physician office visits" (87.5\%). Approximately half of the patients $(50.3 \%)$ had "other outpatient visits," but resources indicative of diagnostic testing (ie, "laboratory" and "radiology") not included in "other outpatient visits" were used by substantially fewer patients. During this period, each patient had a mean (SD) of 15.1 (18.1) outpatient visits to a physician or clinic (median visits 9; interquartile range [IQR] $4,20)$; the number of physician or clinic visits accounted for $73.3 \%$ of all health care visits. While $26.6 \%$ of patients used emergency room services and $14.7 \%$ had hospitalizations, the units of service were low, with 0.8 (2.4) and 0.3 (1.3) visits per patient, respectively. Overall, FM patients visited a health care resource (physician office, clinic, hospital, emergency room, physical therapy, other health care visits), an average of 20.6 (22.6) times (median total health care visits 14; IQR 6,27 ), during the post-index year.

The mean (SD) medication orders per patient was 21.2 (21.5) (median medication orders 16; IQR 7, 29), and encompassed a mean of 11.3 (8.9) unique medications per patient. Among the medications (Figure 2), opioids were the most frequently prescribed medication class, $44.3 \%$ of patients,

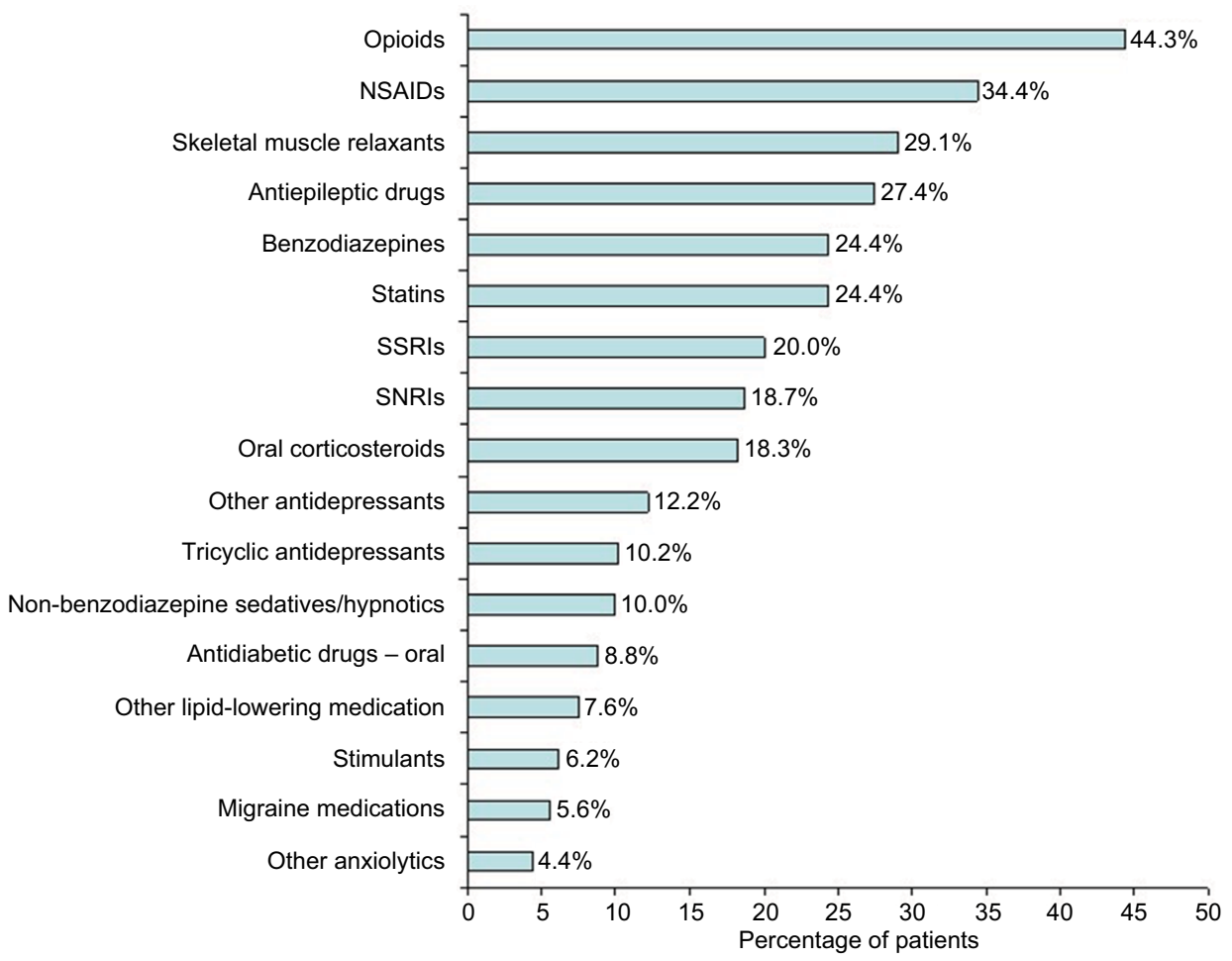

Figure 2 Medications by class prescribed during the 12-month post-index period among patients with fibromyalgia.

Abbreviations: NSAIDs, nonsteroidal anti-inflammatory drugs; SNRIs, serotonin-norepinephrine reuptake inhibitors; SSRIs, selective serotonin reuptake inhibitors. 
followed by nonsteroidal anti-inflammatory drugs in 34.4\%.

Except for statins (24.4\%), most of the drugs with high rates of prescribing were those that are often associated with pain (Figure 2), including anti-epileptics (27.4\%) and a variety of antidepressants, ranging from tricyclic antidepressants $(10.2 \%)$ to selective serotonin reuptake inhibitors $(20.0 \%)$.

When opioids were stratified by those within FM treatment guidelines (ie, weak opioids), just over one-third of the patients $(38.3 \%)$ who were prescribed opioids received weak opioids, representing $16.9 \%$ of the overall population. However, 23,347 patients ( $82.4 \%$ of patients receiving opioid orders) were prescribed opioids not within FM guidelines, although individual patients could be receiving multiple opioids, including those within and not within the guidelines. Patients receiving opioid orders had a mean of 2.8 (3.0) prescriptions for opioids within FM guidelines and 5.8 (7.2) prescriptions for opioids not within guidelines during the 12-months post-index.
The results from negative binomial regression of the total number of all-cause post-index health care visits (Table 3) found the pre-index total number of health care visits to have the greatest association with higher post-index total health care visits (incident rate ratio 1.36). The model further showed that risk of higher health care resource utilization indicated by a higher number of total health care visits was significantly increased by $26 \%(P<0.001)$ among African-Americans (Table 3 ) relative to the comparator group (Caucasians), that is, an incident rate ratio (IRR) indicating 1.26 times as many health care visits. Similarly, there were several comorbid conditions associated with increased number of health care visits, ranging from a $6 \%$ increase for musculoskeletal pain and depression/bipolar disorder (both IRR 1.06 ) to a $21 \%$ increase (IRR 1.21 ) for congestive heart failure (all $P<0.001$ ). Ordering of opioids not within FM guidelines was also associated with a $12 \%$ increase (IRR 1.12) in health care visits relative to those

Table 3 Factors associated with total number of all-cause health care visits during 12-month post-index period

\begin{tabular}{|c|c|c|c|c|}
\hline \multirow[t]{2}{*}{ Factor ${ }^{a}$} & \multirow[t]{2}{*}{ Incident rate ratio } & \multicolumn{2}{|l|}{$95 \% \mathrm{Cl}$} & \multirow[t]{2}{*}{$P$-value } \\
\hline & & Lower & Upper & \\
\hline Total health care visits (pre-index) & 1.36 & 1.33 & 1.39 & $<0.001$ \\
\hline African-American vs Caucasian & 1.26 & 1.23 & 1.29 & $<0.001$ \\
\hline Congestive heart failure & 1.21 & 1.17 & 1.25 & $<0.001$ \\
\hline Coronary heart disease & 1.13 & 1.10 & 1.16 & $<0.001$ \\
\hline Opioids not within FM treatment guidelines & 1.12 & 1.10 & 1.14 & $<0.001$ \\
\hline Gastrointestinal issues $^{b}$ & 1.12 & 1.10 & 1.14 & $<0.001$ \\
\hline Peripheral vascular disease & 1.11 & 1.07 & 1.15 & $<0.001$ \\
\hline Chest pain & 1.09 & 1.07 & 1.11 & $<0.001$ \\
\hline Abdominal pain/cramps & 1.09 & 1.07 & 1.11 & $<0.001$ \\
\hline Depression, bipolar disorder & 1.06 & 1.04 & 1.08 & $<0.001$ \\
\hline Musculoskeletal pain & 1.06 & 1.04 & 1.08 & $<0.001$ \\
\hline Hypertension & 1.06 & 1.04 & 1.08 & $<0.001$ \\
\hline Cerebrovascular disease & 1.06 & 1.03 & 1.08 & $<0.001$ \\
\hline Low back (lumbar) pain & 1.05 & 1.03 & 1.07 & $<0.001$ \\
\hline Confusion or memory problems & 1.04 & 1.02 & 1.07 & 0.003 \\
\hline Migraine, headache & 1.04 & 1.02 & 1.06 & $<0.001$ \\
\hline Rheumatism, excluding back & 1.03 & 1.01 & 1.05 & 0.001 \\
\hline Anxiety-related disorders & 1.03 & 1.01 & 1.05 & 0.006 \\
\hline Osteoarthritis & 1.02 & 1.00 & 1.04 & 0.025 \\
\hline Back or neck pain (non-lumbar) & 1.02 & 1.00 & 1.04 & 0.042 \\
\hline Other outpatient visitsc & 1.00 & 1.00 & 1.00 & $<0.001$ \\
\hline Age & 0.99 & 0.99 & 1.00 & $<0.001$ \\
\hline Unique 3-digit ICD-9 diagnoses & 0.99 & 0.99 & 0.99 & $<0.001$ \\
\hline Sleep disorders & 0.97 & 0.95 & 0.99 & 0.001 \\
\hline Chronic fatigue syndrome & 0.97 & 0.94 & 1.00 & 0.028 \\
\hline Hyperlipidemia & 0.91 & 0.89 & 0.93 & $<0.001$ \\
\hline Emergency room visits & 0.85 & 0.83 & 0.86 & $<0.001$ \\
\hline Inpatient admissions & 0.83 & 0.81 & 0.85 & $<0.001$ \\
\hline
\end{tabular}

Notes: a Factors are measured pre-index. Comorbidities are binary (yes/no) and other factors are counts. ${ }^{\mathrm{b} G a s t r o i n t e s t i n a l ~ i s s u e s ~ i n c l u d e d ~ n a u s e a, ~ v o m i t i n g, ~ d i a r r h e a, ~}$ constipation, loss of appetite. 'Other outpatient visits do not include physician office visits, diagnostic radiology, imaging services, emergency room, or inpatient admissions. Abbreviations: $\mathrm{Cl}$, confidence interval; FM, fibromyalgia; ICD-9, International Classification of Diseases, ninth revision. 
Table 4 Factors associated with total number of medication orders during 12-month post-index period

\begin{tabular}{|c|c|c|c|c|}
\hline \multirow[t]{2}{*}{ Factor $^{\mathrm{a}}$} & \multirow[t]{2}{*}{ Incident rate ratio } & \multicolumn{2}{|l|}{$95 \% \mathrm{Cl}$} & \multirow[t]{2}{*}{$P$-value } \\
\hline & & Lower & Upper & \\
\hline Sex female (vs male) & 1.23 & 1.20 & 1.25 & $<0.001$ \\
\hline Congestive heart failure & 1.12 & 1.08 & 1.17 & $<0.001$ \\
\hline Hypertension & 1.09 & 1.08 & I.II & $<0.001$ \\
\hline Sleep disorders & 1.08 & 1.06 & 1.10 & $<0.001$ \\
\hline Migraine, headache & 1.08 & 1.05 & 1.10 & $<0.001$ \\
\hline Rheumatoid arthritis & 1.07 & 1.04 & I.II & $<0.001$ \\
\hline Depression, bipolar disorder & $\mathrm{I} .07$ & 1.05 & 1.09 & $<0.001$ \\
\hline Anxiety-related disorders & 1.06 & $\mathrm{I} .04$ & 1.09 & $<0.001$ \\
\hline Coronary heart disease & 1.06 & 1.03 & 1.10 & $<0.001$ \\
\hline Peripheral vascular disease & 1.04 & 1.00 & 1.09 & 0.035 \\
\hline Osteoarthritis & 1.04 & 1.02 & 1.06 & $<0.001$ \\
\hline Low back (lumbar) pain & 1.04 & 1.02 & 1.06 & $<0.001$ \\
\hline Irritable bowel syndrome & 1.03 & 1.00 & 1.07 & 0.049 \\
\hline Total health care visits (pre-index) & 1.03 & 1.03 & 1.03 & $<0.001$ \\
\hline Diagnostic radiology, imaging services & 1.02 & 1.01 & 1.03 & $<0.001$ \\
\hline Emergency room visits & 1.01 & 1.00 & 1.01 & $<0.001$ \\
\hline Age & 1.00 & 1.00 & 1.00 & 0.006 \\
\hline Unique 3-digit ICD-9 diagnoses & 0.99 & 0.99 & 1.00 & $<0.001$ \\
\hline Gastrointestinal issues $^{b}$ & 0.97 & 0.95 & 0.99 & 0.001 \\
\hline Hyperlipidemia & 0.96 & 0.95 & 0.98 & $<0.001$ \\
\hline Paresthesias, numbness, tingling & 0.96 & 0.93 & 0.98 & $<0.001$ \\
\hline Dizziness or balance problems & 0.94 & 0.92 & 0.96 & $<0.001$ \\
\hline Musculoskeletal pain & 0.94 & 0.92 & 0.96 & $<0.001$ \\
\hline Other ethnicity (vs Caucasian) ${ }^{c}$ & 0.92 & 0.89 & 0.95 & $<0.001$ \\
\hline
\end{tabular}

Notes: ${ }^{a}$ Factors are measured pre-index. Comorbidities are binary (yes/no) and other factors are counts. ${ }^{b}$ Gastrointestinal issues included nausea, vomiting, diarrhea, constipation, loss of appetite. 'Other ethnicity does not include African-American or Caucasian.

Abbreviations: $\mathrm{Cl}$, confidence interval; ICD-9, International Classification of Diseases, ninth revision.

with orders for opioids within FM guidelines or without any opioid orders $(P<0.001)$.

The results from the negative binomial regression of number of prescriptions (Table 4) showed that the covariate associated with the greatest increase in prescriptions was being female, having an IRR with $23 \%$ more prescriptions than males. As with the factors contributing to overall health care resource utilization, the presence of specific comorbid conditions was also associated with increased medication prescriptions (Table 4).

\section{Discussion}

This analysis provides a practical example of how EHR may be used to characterize health care resource utilization and identify variables associated with high resource use. Consistent with studies using other data sources, this study found FM patients to be frequent users of health care resources and medications. . $^{10,11,14,20,24}$ In this large population of FM patients, whose demographic characteristics were consistent with what may be expected for an FM population (ie, females of primarily middle age), ${ }^{7}$ the primary drivers of all-cause health care resource utilization were "medica- tions ordered" and "physician office visits." These resource categories were also characterized by high units of use per patient in the post-index period. In particular, medication utilization showed that, on average, patients were prescribed 21.2 medication orders for 11.3 different medications during the 12-month post-index period.

This high medication utilization may be related to the fact that these patients had a substantial comorbidity burden. This burden not only consisted of conditions that have been typically reported as being associated with FM, including numerous other musculoskeletal pain conditions and gastrointestinal disorders, but also conditions that have not generally been considered to be associated with FM such as hypertension and hyperlipidemia. ${ }^{3,4}$ The presence of conditions not necessarily associated with FM may not be surprising given the age demographic of this population, and yet other studies have found the same comorbidities in similar percentages of FM patients. ${ }^{6,11,20,24,28,30}$

As has been reported in previous studies of $F M,{ }^{2,10-14}$ opioids were the most frequently prescribed medication class. Notably, the proportion of patients prescribed opioids not mentioned in FM guidelines was approximately twice the 
proportion who were prescribed opioids within guidelines. This is the first analysis to suggest and to quantify that such opioid prescribing may be associated with an increase in resource utilization in these patients. The reason for this association was not determined, but it has been reported that FM patients taking opioids have poorer outcomes than nonopioid users, ${ }^{31}$ which may drive additional health care-seeking behavior. Recent studies have found improved scores for pain severity, pain interference, patient function, insomnia, disability severity, and depression severity in FM patients not taking opioids compared with those taking opioids. ${ }^{15,32}$ A recent observational study reported that the prescribing of opioids after FM diagnoses significantly reduced the likelihood of receiving a guideline-recommended medication. ${ }^{2}$ Our findings of a $12 \%$ increase in health care visits for patients receiving opioids not within FM guidelines provide further evidence of the impact on health care resources in diagnosing and managing this complex condition, from the initial evaluation of patient's need for such opioids to the follow-up and monitoring of their effectiveness.

While antidepressants from various classes were also commonly prescribed, almost half of the patients $(46.8 \%)$ had a diagnosis of depression in the post-index period, so it is likely that at least some antidepressant prescriptions may have been for depression, which is a commonly reported comorbid condition in FM. ${ }^{6,11,14,20,24}$ Reasons for prescribing medications are inconsistently captured in EHR databases due to variations in current data entry practices, ie, providers using EHRs in the US are not required to provide reasons for each medication order and so commonly not entered.

An important observation is that, while use of NLP enabled a search for patients who had documented pain scores, these were inconsistently formatted and reported. Pain severity was documented among only $3.6 \%$ of patients during the 12 months prior to FM diagnosis, despite substantial proportions of patients with pain-related comorbidities such as painful musculoskeletal conditions and headache. While the proportion of patients with pain scores in the post-index period was higher, potentially indicating a greater rate of pain assessment after FM diagnosis, the proportion with a documented pain assessment any time during the 12-month post-index period was nevertheless less than one-third $(30.2 \%)$ of the FM population. Such inconsistent and infrequent documentation makes it difficult to evaluate changes in pain severity over time, and also highlights the likelihood that among patients with this pain condition, the presence and severity of pain may not be regularly evaluated and/or recorded. Whether this lack of pain evaluation is limited to FM or is widespread in other pain conditions is not known, since there are no known studies that have evaluated the frequency of use of pain measures in usual clinical practice among patients with chronic pain conditions. Nonetheless, the low availability of pre-index pain scores indicates that the lack of pain assessment reporting is common.

\section{Limitations}

Although a strength of this study was its use of data derived from EHR, especially since EHRs are now operational in most health systems and with data not otherwise available from other observational sources, the data reported here are subject to potential errors in coding or record keeping at the point of the health care provider, as is the case with all database analyses. Therefore, $\geq 2$ International Classification of Diseases, Ninth Revision codes were used to identify the evaluated population in order to minimize errors in FM coding and to improve the accuracy of identifying the FM cohort. However, it is also possible that there were patients with FM who were excluded from the analysis based on this more conservative inclusion criterion. Furthermore, EHR datasets only document the order by the health care provider and are not confirmatory that the order was followed. This limitation may be especially relevant for pharmacotherapy; medications ordered by the health care provider and documented in the EHR may not necessarily have been filled or administered to patients. Another limitation is that since resource utilization was regardless of cause, no implications can be made as to what extent the resources used were associated directly with FM. In this regard, since many of these patients had comorbidities, the prescribing of a specific medication could have been for a condition other than FM.

\section{Conclusion}

Using data from EHR, substantial all-cause health care resource utilization was observed among FM patients. Physician office visits and pharmacotherapy were identified as the two main drivers of all-cause health care resource utilization, and opioids were the most frequently prescribed medication class overall. While two demographic factors (female and African-American) were associated with high resource use, the majority of variables associated with greater health care resource utilization were comorbid conditions representing a variety of organ systems. However, prescribing of opioids was also associated with greater health care resource utilization. These results suggest that EHR can be a useful tool for identifying patients with high health care resource utilization. 
Such identification represents an important first step in determining appropriate strategies for potentially managing patients with FM with a goal of improving patient outcomes and reducing medical costs.

\section{Acknowledgments}

The authors gratefully acknowledge the important contributions of Douglas Weldon of Truven Health Analytics and Erin Thomson, formerly of Truven Health Analytics, for their roles in the research design and analysis of data.

This study was sponsored by Pfizer. Editorial/medical writing support was provided by E Jay Bienen and was funded by Pfizer.

\section{Disclosure}

Elizabeth T Masters, Joseph C Cappelleri, and Steven Faulkner are employees and stockholders of Pfizer. Jay M Margolis and David M Smith are employees of Truven Health Analytics which was paid by Pfizer in connection with the development of this manuscript. The authors report no other conflicts of interest in this work.

\section{References}

1. Clauw DJ, Arnold LM, McCarberg BH; FibroCollaborative. The science of fibromyalgia. Mayo Clin Proc. 2011;86(9):907-911.

2. Halpern R, Shah SN, Cappelleri JC, Masters ET, Clair A. Evaluating guideline-recommended pain medication use among patients with newly diagnosed fibromyalgia. Pain Pract. 2016;16(8):1027-1039.

3. Wolfe F, Clauw DJ, Fitzcharles M-A, et al. The American College of Rheumatology preliminary diagnostic criteria for fibromyalgia and measurement of symptom severity. Arthritis Care Res. 2010;62(5):600-610.

4. Bennett RM. Clinical manifestations and diagnosis of fibromyalgia. Rheum Dis Clin North Am. 2009;35(2):215-232.

5. Vincent A, Lahr BD, Wolfe F, et al. Prevalence of fibromyalgia: a population-based study in Olmsted County, Minnesota, utilizing the Rochester Epidemiology Project. Arthritis Care Res (Hoboken). 2013;65(5):786-792.

6. Walitt B, Nahin RL, Katz RS, Bergman MJ, Wolfe F. The prevalence and characteristics of fibromyalgia in the 2012 National Health Interview Survey. PLoS One. 2015;10(9):e0138024.

7. Wolfe F, Ross K, Anderson J, Russell IJ, Hebert L. The prevalence and characteristics of fibromyalgia in the general population. Arthritis Rheum. 1995;38(1):19-28.

8. Carville SF, Arendt-Nielsen S, Bliddal H, et al. EULAR evidence based recommendations for the management of fibromyalgia syndrome. Ann Rheum Dis. 2008;67(4):536-541.

9. Fitzcharles MA, Ste-Marie PA, Goldenberg DL, et al; National Fibromyalgia Guideline Advisory Panel. 2012 Canadian Guidelines for the diagnosis and management of fibromyalgia syndrome: executive summary. Pain Res Manag. 2013;18(3):119-126.

10. White LA, Robinson RL, Yu AP, Kaltenboeck A, Samuels S, Mallett D, Birnbaum HG. Comparison of health care use and costs in newly diagnosed and established patients with fibromyalgia. J Pain. 2009;10(9):976-983.

11. Berger A, Sadosky A, Dukes EM, Edelsberg J, Zlateva G, Oster G. Patterns of health care utilization and cost in patients with newly diagnosed fibromyalgia. Am J Manag Care. 2010;16(Supp1):S126-S137.

12. McNett M, Goldenberg D, Schaefer C, Hufstader M, Baik R, Chandran A, Zlateva G. Treatment patterns among physician specialties in the management of fibromyalgia: results of a cross-sectional study in the United States. Curr Med Res Opin. 2011;27(3):673-683.
13. Bernauer M, Wu N, Chen SY, Peng X, Boulanger L, Zhao Y. Use of select medications prior to duloxetine initiation among commerciallyinsured patients. J Pain Res. 2015;5:271-278.

14. Schaefer C, Mann R, Masters ET, et al. The comparative burden of chronic widespread pain and fibromyalgia in the United States. Pain Pract. 2016;16(5):565-579.

15. Peng X, Robinson RL, Mease P, et al. Long-term evaluation of opioid treatment in fibromyalgia. Clin J Pain. 2015;31(1):7-13.

16. Painter JT, Crofford LJ. Chronic opioid use in fibromyalgia syndrome: a clinical review. J Clin Rheumatol. 2013;19(2):72-77.

17. Fitzcharles MA, Ste-Marie PA, Goldenberg DL, et al. 2012 Canadian guidelines for the diagnosis and management of fibromyalgia syndrome. Available from: http://fmguidelines.ca/?page_id=21. Accessed April 8, 2016.

18. Hoffman DL, Dukes E. The health status burden of people with fibromyalgia: a review of studies that assessed health status with the SF-36 or the SF-12. Int J Clin Pract. 2008;62(1):115-126.

19. Luo X, Cappelleri JC, Chandran A. The burden of fibromyalgia: assessment of health status using the EuroQol (EQ-5D) in patients with fibromyalgia relative to other chronic conditions. Health Outcomes Res Med. 2011;2(4):e203-e214.

20. Schaefer C, Chandran A, Hufstader M, et al. The comparative burden of mild, moderate and severe fibromyalgia: results from a cross-sectional survey in the United States. Health Qual Life Outcomes. 2011;9(1):71.

21. McDonald M, DiBonaventura MD, Ullman SJP. Musculoskeletal pain in the workforce: the effects of back, arthritis and fibromyalgia pain on quality of life and work productivity. J Occup Environ Med. 2011; 53(7):765-770.

22. Knight T, Schaefer C, Chandran A, Zlateva G, Winkelmann A, Perrot S. Health-resource use and costs associated with fibromyalgia in France, Germany, and the United States. Clinicoecon Outcomes Res. 2013;5:171-180.

23. Masters ET, Mardekian J, Emir B, Clair A, Kuhn M, Silverman S. Electronic medical record data to identify variables associated with a fibromyalgia diagnosis: importance of health care resource utilization. J Pain Res. 2015;8:131-138.

24. Chandran A, Schaefer C, Ryan K, Baik R, McNett M, Zlateva G. The comparative economic burden of mild, moderate, and severe fibromyalgia: results from a retrospective chart review and a cross-sectional survey of working-age U.S. adults. J Manag Care Pharm. 2012;18(6): 415-426.

25. Hayrinen K, Saranto K, Nykanen P. Definition, structure, content, use and impacts of electronic health records: a review of the research literature. Int J Med Inform. 2008;77(5):291-304.

26. Wells AF, Arnold LM, Curtis CE, Dunegan LJ, Lapp CW, McCarberg BH, Clair A. Integrating health information technology and electronic health records into the management of fibromyalgia. Postgrad Med. 2013;125(4):70-77.

27. Emir B, Masters ET, Mardekian J, Clair A, Kuhn M, Silverman SL. Identification of a potential fibromyalgia diagnosis using random forest modeling applied to electronic medical records. J Pain Res. 2015;8: 277-288.

28. Gore M, Tai KS, Chandran A, Zlateva G, Leslie D. Clinical comorbidities, treatment patterns, and health care costs among patients with fibromyalgia newly prescribed pregabalin or duloxetine in usual care. J Med Econ. 2012;15(1):19-31.

29. Dobson A, Barnett A. An Introduction to Generalized Linear Models. 3rd ed. Boca Raton, FL: Chapman \& Hall; 2008.

30. Vincent A, Whipple MO, McAllister SJ, Aleman KM, St Sauver JL. A cross-sectional assessment of the prevalence of multiple chronic conditions and medication use in a sample of community-dwelling adults with fibromyalgia in Olmsted County, Minnesota. BMJ Open 2015;5:e006681.

31. Goldenberg DL, Clauw DJ, Palmer RE, Clair AG. Opioid use in fibromyalgia: a cautionary tale. Mayo Clin Proc. 2016;91(5):640-648.

32. Fitzcharles MA, Faregh N, Ste-Marie PA, ShirY. Opioid use in fibromyalgia is associated with negative health related measures in a prospective cohort study. Pain Res Treat. 2013;2013:898493. 
ClinicoEconomics and Outcomes Research is an international, peerreviewed open-access journal focusing on health technology assessment, pharmacoeconomics and outcomes research in the areas of diagnosis, medical devices, and clinical, surgical and pharmacological intervention. The economic impact of health policy and health systems organization also constitute important areas of coverage. The manuscript management system is completely online and includes a very quick and fair peer-review system, which is all easy to use. Visit http://www.dovepress.com/testimonials.php to read real quotes from published authors.

Submit your manuscript here: https://www.dovepress.com/clinicoeconomics-and-outcomes-research-journal 